\title{
Contrasting Transcriptional Programs Control Postharvest Development of Apples (Malus $x$ domestica Borkh.) Submitted to Cold Storage and Ethylene Blockage
}

Tatiane Timm Storch, ${ }^{\dagger \dagger}$ Taciane Finatto, ${ }^{\dagger, \perp}$ Maryline Bruneau, ${ }^{\S}$ Mathilde Orsel-Baldwin, ${ }^{\S}$ Jean-Pierre Renou, ${ }^{\S}$ Cesar Valmor Rombaldi, ${ }^{\ddagger}$ Vera Quecini, ${ }^{\dagger}$ François Laurens, ${ }^{\S}$ and César Luis Girardi*,†®

\author{
${ }^{\dagger}$ Embrapa Uva e Vinho, Bento Gonçalves, RS 95701-008, Brazil \\ ${ }^{\ddagger}$ Departamento de Ciência e Tecnologia Agroindustrial, Faculdade de Agronomia Eliseu Maciel, Universidade Federal de Pelotas, \\ Pelotas, RS 96050-500, Brazil \\ ${ }^{\S}$ Bâtiment B, Institut de Recherche en Horticulture et Semences IRHS, Institut National de la Recherche Agronomique INRA, 49071 \\ Beaucouzé, France
}

Supporting Information

ABSTRACT: Apple is commercially important worldwide. Favorable genomic contexts and postharvest technologies allow yearround availability. Although ripening is considered a unidirectional developmental process toward senescence, storage at low temperatures, alone or in combination with ethylene blockage, is effective in preserving apple properties. Quality traits and genome wide expression were integrated to investigate the mechanisms underlying postharvest changes. Development and conservation techniques were responsible for transcriptional reprogramming and distinct programs associated with quality traits. A large portion of the differentially regulated genes constitutes a program involved in ripening and senescence, whereas a smaller module consists of genes associated with reestablishment and maintenance of juvenile traits after harvest. Ethylene inhibition was associated with a reversal of ripening by transcriptional induction of anabolic pathways. Our results demonstrate that the blockage of ethylene perception and signaling leads to upregulation of genes in anabolic pathways. We also associated complex phenotypes to subsets of differentially regulated genes.

KEYWORDS: 1-methylcyclopropene, firmness, fruit quality, gene expression, microarray

\section{INTRODUCTION}

Apple (Malus $x$ domestica Borkh) economic importance is largely dependent on the ability to extend its window of availability using several postharvest conservation strategies. As other climateric fruits, apple has good storability under low temperatures, especially in combination with the inhibition of ethylene perception, retaining high consumer acceptance for up to ten months, depending on the cultivar. ${ }^{1,2}$ From the commercial standpoint, effective postharvest techniques should result in the conservation of the sensory characteristics of fresh fruits after months of storage. Moreover, as functional foods, it is critical that the nutraceutical properties of fruits remain unaltered during and after conservation. In apple, the quality to the consumer is largely determined by flesh firmness, contents of soluble solids, and acidity, ${ }^{3}$ driving extensive efforts to identify and functionally characterize the genes associated with the traits. ${ }^{2,4-6}$ Currently, the transcriptional repression of ripening and senescence pathways during conservation is considered crucial to maintain the fruit quality attributes after harvest. $^{2,7-9}$

Plant postembryonic development is highly plastic, ${ }^{10}$ although flowering, fruit ripening, organ abscission, and senescence are frequently considered unidirectional processes. ${ }^{11}$ The gaseous hormone ethylene has a major role in controlling these processes, along with its function in stimulating or inhibiting vegetative growth depending on developmental and environmental conditions. ${ }^{12}$ Quality traits in fruits undergo extensive modifications during ripening and afterward, including the conversion of starch to soluble sugars, synthesis of volatile aroma compounds, and decrease of acidity and flesh firmness. ${ }^{2}$ Although some of these attributes, such as flesh firmness and aroma, are clearly dependent on ethylene, others like acidity and soluble solids content are still under unknown regulation. ${ }^{13,14}$ Ethylene mediated transduction pathways are also involved in the integration of biotic and abiotic stress signals. ${ }^{12}$ The competitive inhibitor 1-methylcyclopropene (1-MCP) is frequently used after harvest to extend the shelf life of apples by suppressing the hormone perception and its autocatalytic biosynthesis and signaling. ${ }^{15}$ In the fruit perspective, the application of postharvest conservation techniques, such as low temperatures and ethylene perception inhibition, consist of a complex scenario that requires the integration of endogenous developmental processes toward senescence, with responses to the extreme environmental conditions used during storage. Thus, it is not

Received: March 28, 2017

Revised: August 2, 2017

Accepted: August 3, 2017

Published: August 3, 2017 
A

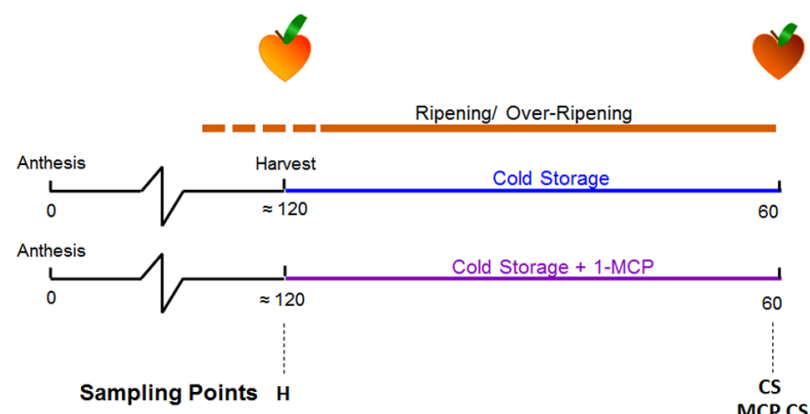

B

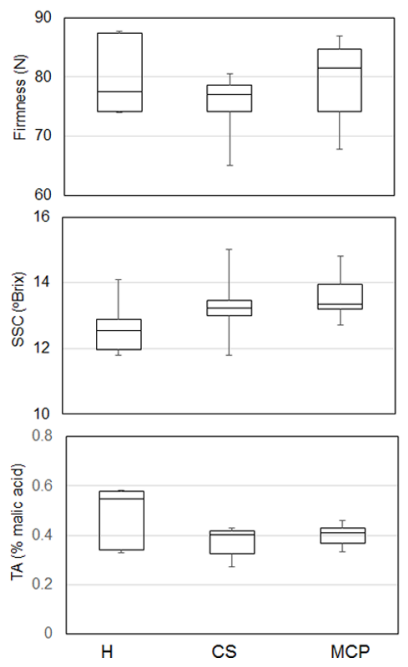

C

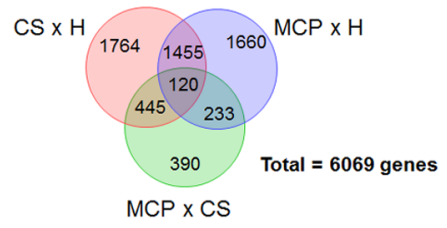

D

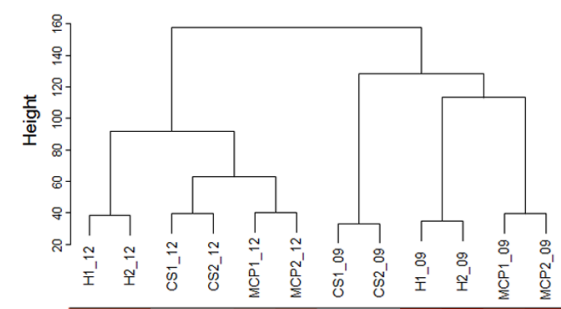

Fsc ( ${ }^{\circ} \mathrm{Br}$ rix $)$

TA (\% malic acid)

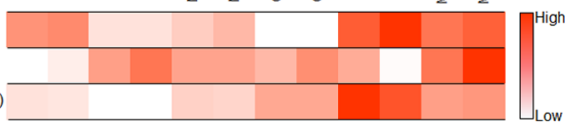

Figure 1. Schematic representation of the sampling points and the apple ripening and postharvest conservation timeline (A). Apple quality traits flesh firmness (F), soluble solids contents (SSC), and titratable acidity (TA) at harvest (H) and after 60 days of cold storage alone (CS) and with 1MCP application (MCP) (B). Summary graphic representation of the transcriptional profile of apples at $\mathrm{H}$ and after CS and MCP, representing the relationship of the differentially expressed genes (DEGs) among the treatments (C) and hierarchical clustering analysis (HCA) of the samples associated with a heatmap representation of the quality traits (D).

surprising that conservation techniques are often associated with undesirable physiological conditions, such as mealiness and phenolic browning. 6,16

Although genetic and genomic data are largely available on the initial responses to postharvest technologies, integrative studies on the later stages of ripening of apples submitted to 1MCP treatment under low temperature remain scarce.

The current work aimed to provide the transcriptional framework underlying the complex phenotypes of 'Gala' associated with consumer acceptance at harvest and after application of conservation techniques, storage at low temperatures alone and in combination with ethylene perception blockage, for 60 days. We hypothesized that the identification of gene expression networks and their association with quality traits may provide further insights in the control of complex sensory characteristics and the effect of external conservation factors on the endogenous mechanisms regulating postharvest ripening.

\section{MATERIALS AND METHODS}

Plant Material and Postharvest Treatments. Apples (Malus $x$ domestica Borkh.) from cultivar Gala, clone Baigent, were collected at the commercial maturity stage from commercial orchards in the State of Rio Grande do Sul, Brazil, as two biological replicates (harvesting seasons of 2009 and 2012). Physiological and genome-wide expression analyses were performed at harvest $(\mathrm{H})$ and after 60 days under cold storage (CS) at $0 \pm 0.5{ }^{\circ} \mathrm{C}(90 \% \pm 5 \%$ relative humidity $)$ in the absence or presence of 1-methylcyclopropene (MCP). Ethylene inhibitor treatment was carried out immediately after harvest with 1 $\operatorname{ppm}\left(1 \mu \mathrm{g} \cdot \mathrm{L}^{-1}\right)$ of $1-\mathrm{MCP}$ for $24 \mathrm{~h}$ in a $370 \mathrm{~L}$ chamber containing 50 $\mathrm{kg}$ of apples at room temperature $\left(\mathrm{RT}=20 \pm 0.5{ }^{\circ} \mathrm{C}, 80 \% \pm 5 \%\right.$ relative humidity). After CS and $\mathrm{MCP}$, the fruits were kept for 7 days at RT to simulate the marketing period before evaluation of flesh firmness (F), soluble solids content (SSC), and titratable acidity (TA).

Physiological Analyses. Quality traits sweetness, acidity, and texture were evaluated as soluble solids content (SSC), titratable acidity (TA), and flesh firmness, respectively, as described in ref 2 . The parameters were determined from three biological replicates, consisting of five randomly picked fruits per harvesting season (2009 and 2012). Statistical analyses consisting of ANOVA and Tukey test were conducted using the package Agricolae in $\mathrm{R}^{17}$

RNA Extraction and Microarray Transcriptional Profiling. Total RNA was extracted as described ${ }^{18}$ with minor modifications, described in refs 2 and 19, from $6 \mathrm{~g}$ of frozen flesh obtained from a pool of five representative fruits at each sampling point from 2009 and 2012 (Figure 1A). Total RNA was quantified spectrophotometrically using NanoDrop ND-1000 (Thermo Scientifics, Wilmington, DE, USA), and the integrity was confirmed by agarose gel electrophoresis.

Purified mRNAs were amplified, labeled, and cohybridized according to ref 20 as follows: aRNAs were produced with Message AmpII aRNA amplification kit (Ambion Invitrogen, Waltham, MA, USA) from $200 \mathrm{ng}$ of total RNA. Then, $5 \mu \mathrm{g}$ of each aRNA were retrotranscribed and labeled with either cyanine-3 (Cy3) or cyanine-5 (Cy5) fluorescent dye (Interchim, Montluçon, France). Labeled samples were combined with $30 \mathrm{pmol}$ of each dye and cohybridized to NimbleGen microarray AryANE v1.0 containing 126022 60-mer oligonucleotide probes. ${ }^{20}$ Deva software (NimbleGen Roche, Madison, WI, USA) was used to extract pair-data files from the scanned images, obtained using the MS200 microarray scanner (NimbleGen Roche, Madison, WI, USA). 


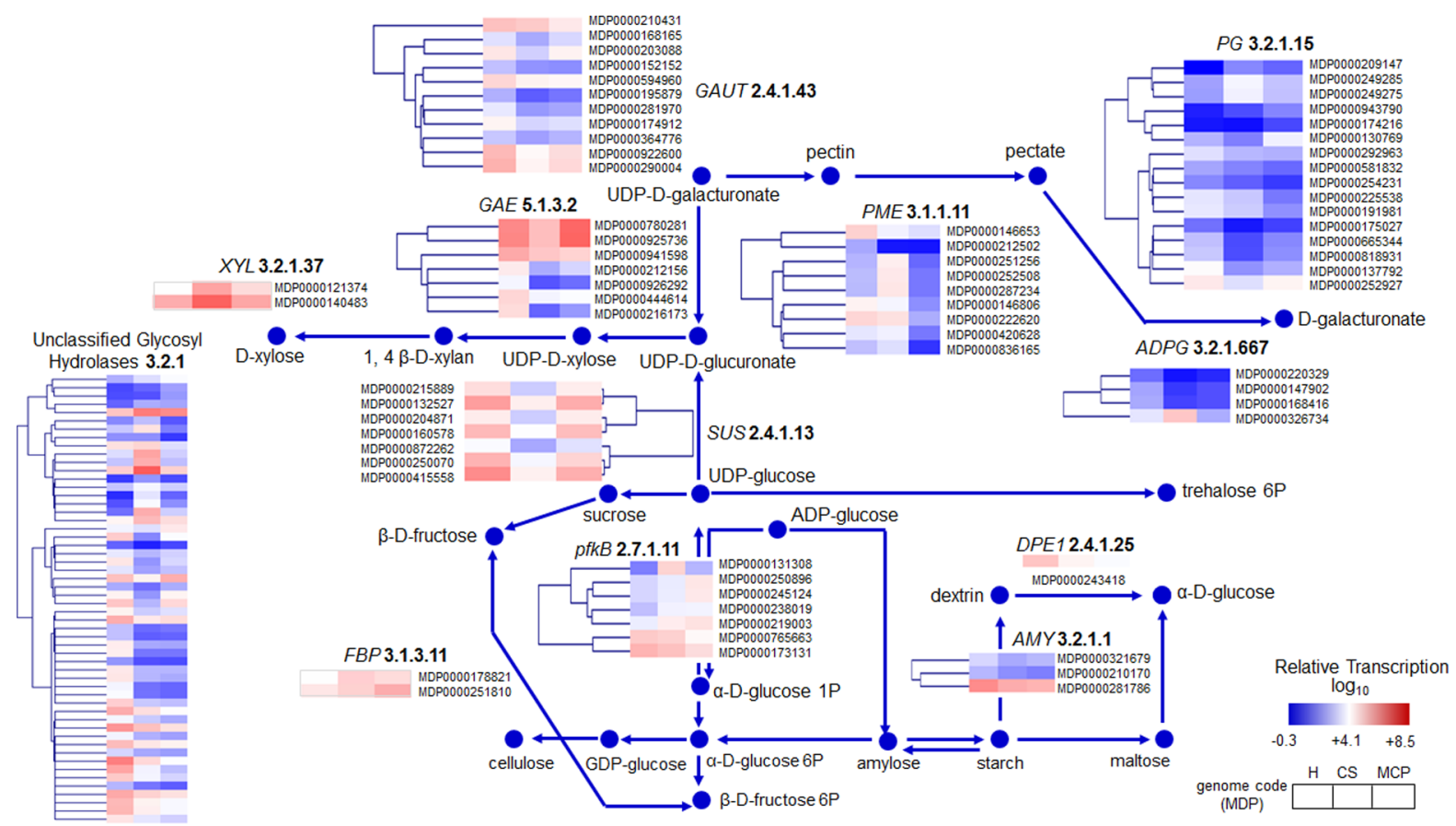

Figure 2. Schematic representation of the transcriptional profile of the DEGs associated with starch and sucrose metabolism (KEGG map 00500 and $00520)$ at harvest $(\mathrm{H})$ and after 60 days of cold storage alone (CS) and with 1-MCP application (MCP). Enzyme coding sequences are represented by abbreviation names and Enzyme Commission number (EC). Relative transcription levels are shown as heatmap, and represent means between 2009 and 2012 samples. HCA was performed based on functional characterization of the DEGs, using Spearman Rank correlation as distance metric, and complete linkage clustering.

Samples at harvest $(\mathrm{H})$ and from cold storage $(\mathrm{CS})$ and 1-MCP treatment $(\mathrm{MCP})$ were interrelated in three comparisons $(\mathrm{CS} \times \mathrm{H}$; $\mathrm{MCP} \times \mathrm{H} ; \mathrm{MCP} \times \mathrm{CS})($ Figure $1 \mathrm{~B})$. Reference RNA was hybridized along with RNA from the treatments using the opposite dye. For each comparison, two independent biological replicates were used with control and experimental cDNA clones labeled with $\mathrm{Cy} 3$ and $\mathrm{Cy} 5$, respectively. The dye-swap was included to eliminate possible bias from fluorescent dyes.

For each comparison, four microarrays were hybridized, as two independent biological replicates consisting of samples from 2009 and 2012 and two dye-swap technical replicates, totaling 12 arrays.

Differential Gene Expression Analyses. All statistical analyses were conducted as described ${ }^{20}$ using $\mathrm{R}$ language. ${ }^{21}$ Briefly, fluorescence intensity data were normalized using the Lowess method, and differential expression analyses were carried out using the $\mathrm{mmFit}$ function and the Bayes moderated $t$ test using the limma package. ${ }^{22}$ The normalized values were corrected for the background to estimate gene expression levels. Significant differential gene expression was determined by false discovery rate (FDR) $p$-values after BenjaminiHochberg $(\mathrm{BH})$ correction $<0.1$. Microarray data is available at Gene Expression Omnibus (http://www.ncbi.nlm.nih.gov/geo/) under the accession number GSE59728.

Further analyses were performed using the sense probes on the array. Differentially expressed probe sequences from AryANE 12X 135 $\mathrm{K}$ chip $^{20}$ were validated against apple genome assembly v.1.0p and v.3.0al contigs at GDR (Genome Database for Rosaceae - http://www. rosaceae.org/) and by maximum likelihood phylogenetic analyses with functionally characterized Arabidopsis proteins. Sequences failing to retrieve coherent transcripts and deduced proteins or phylogenetically divergent from their counterparts in model species were eliminated from the analyses.

Gene module identification and correlation with physiological data were performed employing the package WGCNA ${ }^{23}$ in R. Co-regulated gene modules were identified using a stepwise network construction mode based on coexpression similarity, calculated from adjacency data elevated to soft thresholding power $(\beta)$ that generates a tree with approximate scale-free topology (fit index $=94 \%$ ), considering a minimum module size with 30 genes. Modules with highly similar expression profiles (correlation $\geq 75 \%$ ) were merged by quantifying the coexpression similarity of entire modules using clustering analyses of the correlations between the Eigengenes in each module. ${ }^{23}$

Functional and Relational Analyses of Differentially Expressed Genes (DEGs). Genes exhibiting significant differential expression were classified by gene ontology (GO) analyses and metabolic pathways, according to the Mapman v.3.6.0 RC1 scheme. ${ }^{24}$

Gene ontology enrichment analyses were performed by a hypergeometric test with 0.05 significance threshold after $\mathrm{BH}$ corrected FDR $p$-values using agriGO ${ }^{25}$ and summarized based on measures of semantic similarity using REVIGO ${ }^{26}$ in R. Detailed metabolite pathways and gene expression profiles were generated using the KEGGScape plugin ${ }^{27}$ in Cytoscape 3.4.

Authority and hub scores of the nodes (genes) in each network were calculated as the principal eigenvector of $\mathbf{t}(\mathbf{A}) * \mathbf{A}$ and $\mathbf{A}^{*} \mathbf{t}(\mathbf{A})$, respectively, where $\mathbf{A}$ is the adjacency matrix of the graph, using the package igraph ${ }^{28}$ in $\mathrm{R}$.

Gene Expression Validation by RT-qPCR. Microarray expression data were validated by RT-qPCR for 14 genes chosen based on their expression profile and putative biological function. Primers were designed based on the coding sequences (CDs) of Malus $x$ domestica genome using the software Primer3Plus, ${ }^{29}$ to generate amplicons ranging from 80 to 150 base pairs (Table S3). Synthesis of cDNA and qPCR conditions were performed as described. ${ }^{19}$ Results were normalized employing the reference gene $M d U B I$ and the genes corresponding to apple genome identifiers MDP0000217860 and MDP0000271281, which showed stable transcription in the microarray analyses. Expression profile validation for 14 genes is shown in Figure S6.

\section{RESULTS}

Fruit Quality Changes after Harvest. To gain insight into the physiological changes of 'Gala' destined to conservation before marketing, we sampled fruits from commercial orchards in two harvesting seasons (2009 and 2012) and characterized 


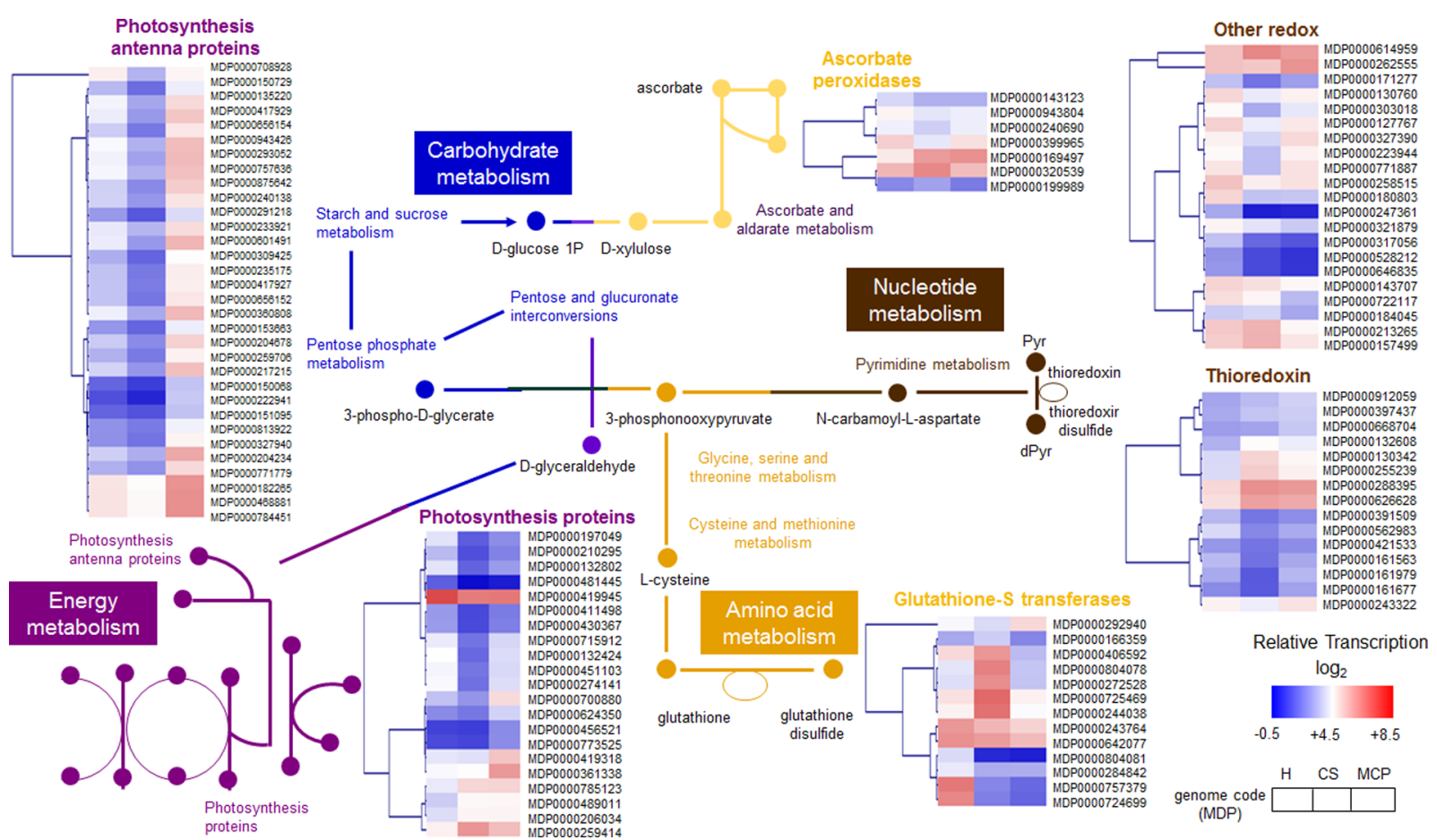

Figure 3. Schematic representation of the transcriptional profile of the DEGs associated with metabolic pathways (KEGG map 01100) at harvest $(\mathrm{H})$ and after 60 days of cold storage alone (CS) and with 1-MCP application (MCP). Relative transcription levels are shown as heatmap and represent means between 2009 and 2012 samples. HCA was performed based on functional characterization of the DEGs, using Spearman Rank correlation as distance metric, and complete linkage clustering.

attributes associated with quality at harvest and after 60 days of $\mathrm{CS}$ in the presence and absence of 1-MCP treatment (Figure 1A). The prevalent quality attributes investigated were sweetness, evaluated as SSC, acidity, as TA, and texture, as flesh firmness (F) (Figure 1B,D).

The studied quality traits responded differently to conservation techniques, with greater effects observed for F (Figure 1B). After two months, the storage treatments CS and MCP did not fully prevent the progression of acidity and firmness loss, and sugar accumulation (Figure 1B). The combined treatment $(\mathrm{MCP})$ had greater impact on firmness, retaining approximately $92 \%$ of flesh consistency (Figure 1B). Physiological differences due to environmental conditions between the harvesting seasons influenced harvest maturity and the amplitude of minimum and maximum value of quality traits (Figure 1B).

Genome Wide Transcriptional Profile. A total of 6069 genes $(9.63 \%$ of the total) exhibited significant differential regulation among the treatments (Figure 1C). Pairwise analyses of the DEGs demonstrated the prevalence of development on differential gene regulation (93.5\%) in comparison to CS and MCP, whereas inhibition of ethylene perception and signaling alone affected the transcription of $6.4 \%$ (Figure 1C). Environmental factors affected the transcriptional profile, although treatment samples still exhibit smaller Euclidian distance within the harvesting seasons (Figure 1D).

Gene ontology classification demonstrated significant enrichment in anabolic and catabolic processes, associated with oxidation-reduction maintenance, energy production, carbohydrate metabolism, transport, among others (Figure S1, Figure S2). Functional classification of the DEGs demonstrated association with carbohydrate metabolism (Figure 2), cellular redox homeostasis, and energy production (Figure 3). Many hydrolase coding sequences $(0.84 \%, 34 / 4044)$ were differentially regulated in response to the tested treatments, including glycosyl hydrolases and pectin methylesterases (Figure 2, Figure 3). Xylosidase-like (EC 3.2.1.37, XYL) transcripts exhibit two profiles, with a cluster being induced after harvest (MDP0000121374) and induced by cold storage (MDP0000140483) (Figure 2). The regulation of pectin methylesterase-like (E.C. 3.1.1.11, PME) genes exhibited three distinct profiles, mainly differing with their transcription level at $\mathrm{H}$ and in response to MCP (Figure 2).

Transcripts of pectic acid hydrolyzing enzymes were also differentially regulated; $C S$ and MCP repressed the transcription of exopoly- $\alpha$-galacturonase (EC 3.2.1.667, ADPG) like sequences (MDP0000220329, MDP0000147902, MDP0000168416). Transcripts coding for polygalacturonase (EC 3.2.1.15, PG) like proteins exhibited a bimodal transcription pattern in response to conservation, with a subset being induced and the other repressed under CS and MCP (Figure 2). In contrast, the transcription of genes coding for enzymes associated with the biosynthesis of sucrose from photosynthesis, sucrose synthase (EC 2.4.1.13, SUS), was repressed by CS (Figure 2). The transcription of genes showing similarity to sequences coding for enzymes of starch catabolism, such as $\alpha$-amylase (EC 3.2.1.1, AMY) and 4- $\alpha$-glucanotransferase (EC 2.4.1.25, DPE1 - DISPROPORTIONATING ENZYME1), was affected during storage, with a repression of the DPE1 (MDP0000243418) and AMY-like genes (MDP0000210270, MDP0000321679, MDP0000281786) by CS and MCP (Figure 2).

Gene families coding for deduced proteins with similarity to enzymes associated with cell wall subunit biosynthesis, such as UDP-D-glucuronate 4-epimerase (EC 5.1.3.6, GAE) and polygalacturonate 4 - $\alpha$-galacturonosyltransferase (EC 2.4.1.75, 
A
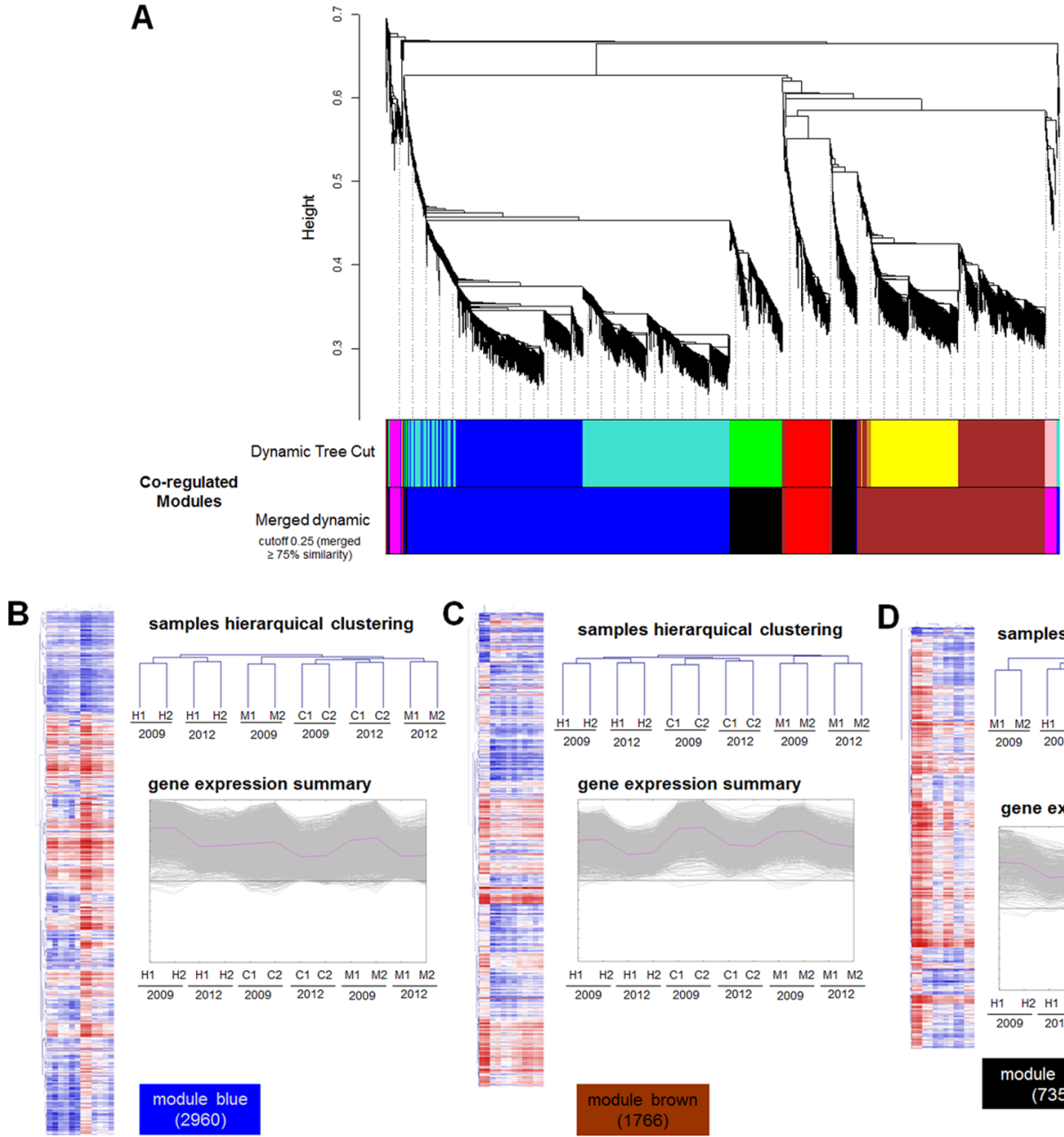

D
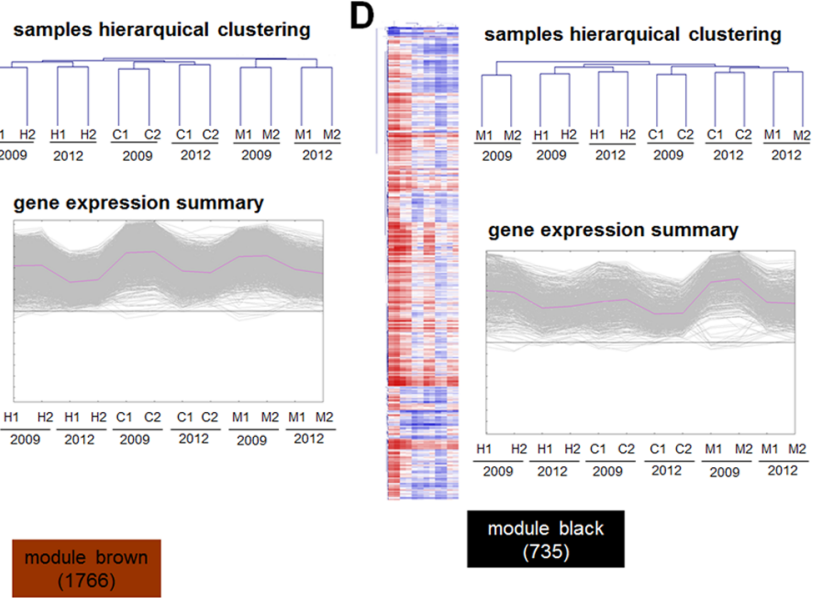

$\mathbf{E}$
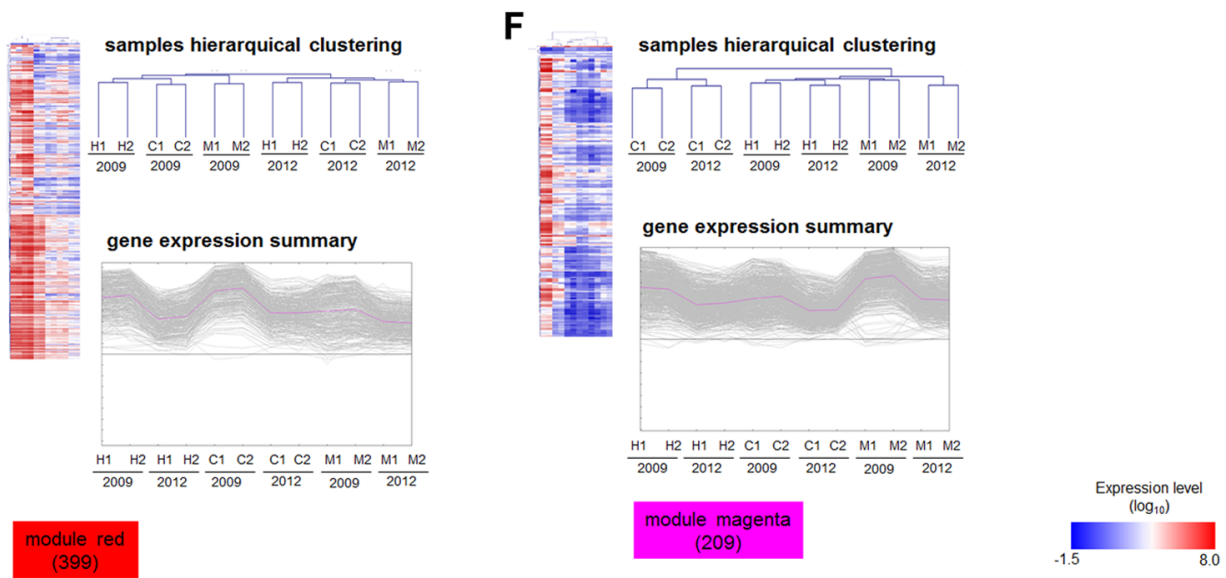

Figure 4. Dendrogram representation of clustering of the DEGs, at harvest $(\mathrm{H})$ and after 60 days of cold storage alone (C) and with 1-MCP application (M) in 2009 and 2012, employing dissimilarity based on topological overlap and the assigned co-regulated modules represented by colors (A), expression summary and HCA of genes and samples in modules blue (B), brown (C), black (D), red (E), and magenta (F).

GAUT) also exhibited complex regulatory patterns, with two distinct regulation profiles of GAE- and three of GAUT-like sequences (Figure 2).

The expression level of ascorbate peroxidases was high for all investigated conditions (Figure 3). Most of the genes coding for transcripts with similarity to photosynthesis antenna proteins were repressed by CS and induced by ethylene blockage (MCP), whereas the transcription of sequences coding for other photosynthesis associated proteins, such as oxygen evolving complexes, exhibited two principal distinct profiles in response to CS and MCP (Figure 3).

Coexpressed Gene Module Identification and Their Correlation to the Quality Traits. Five co-regulated gene modules, designated by colors blue, brown, black, red, and magenta, were identified (Figure 4A). The number of genes per module ranged from 2960 (48.8\%, module blue) to 209 (3.4\%, module magenta) (Figure 4, Table S2). The weight of the association between each gene in the modules is represented as 
A

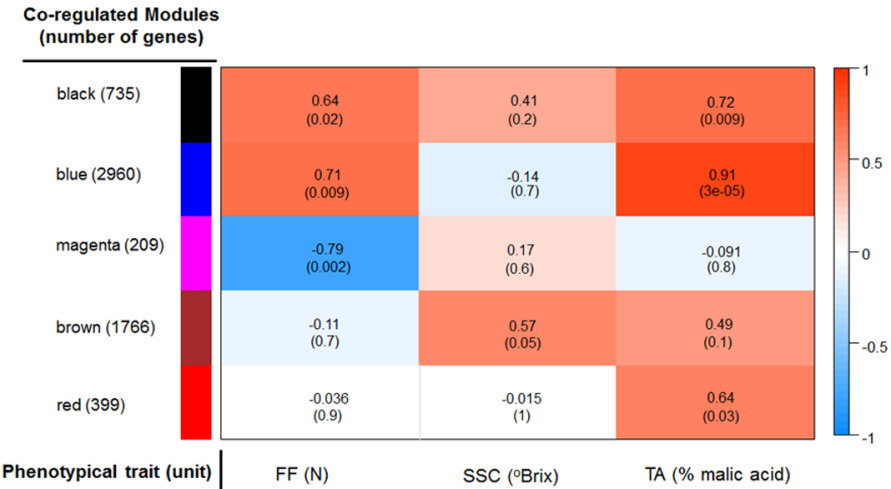

B
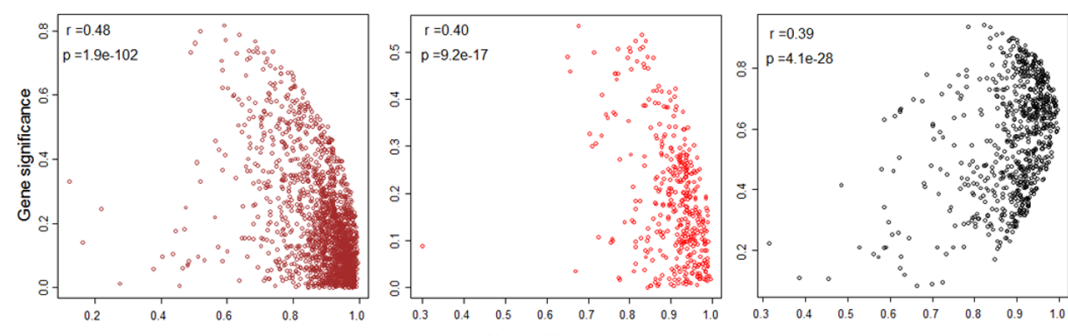

C
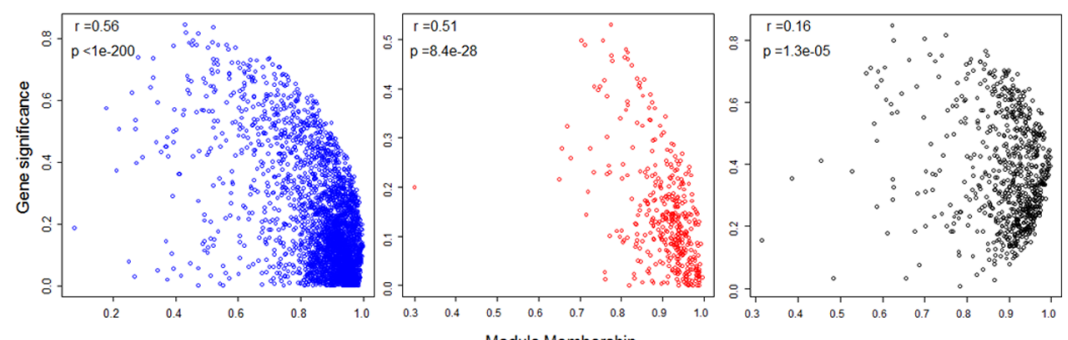

D
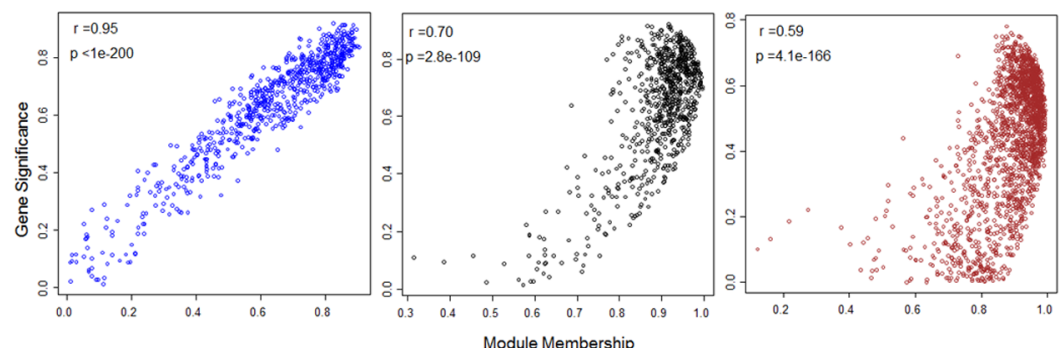

Figure 5. Correlation analysis between the module Eigengene and apple quality traits (A). Pearson's $r$ and $p$-value are shown in each cell, colored as a heatmap based on the correlation strength. The modules are represented as lines and quality traits as columns. Correlation analyses between Gene Significance (GS) and Module Membership (MM) for the modules with higher association to flesh firmness (N) (B), soluble solids contents ( ${ }^{\circ}$ Brix) (C), and titratable acidity (\% malic acid) (D).

heatmap in Figure S5. Transcripts in larger modules blue and brown exhibited distinct expression patterns at $\mathrm{H}$, clustered with MCP (2009) or CS (2009 and 2012) in blue and brown, respectively (Figure 4B,C). Expression summary in module brown demonstrates the influence of the harvesting season on the transcriptional profile (Figure 4C). The transcription of genes in module black is characterized by a marked response to ethylene blockage in 2009, and a closer similarity in the profile in response to CS (2009 and 2012) and to MCP in 2012 (Figure 4D). The most important factor controlling gene expression in module red was harvesting season, with the transcriptional profiles clustered according to the year, with generally higher levels of transcription in 2009 for all tested conditions (Figure 4E). The low temperatures during storage
(CS) had a prevalent effect on the transcription of genes in module magenta (Figure 4F).

To identify important genes associated with sensory traits, we have quantified module-trait associations using the Eigengene profile for each module. Module blue exhibited high positive correlation to acidity (91\%) and firmness (71\%), whereas module magenta exhibited a high negative correlation to firmness (79\%) (Figure 5A). Modules brown and black were positively correlated to soluble solids, $57 \%$ and $41 \%$, respectively, although with higher $p$-values (Figure 5A). Similarly, the positive correlations between fruit acidity and modules brown and red, respectively, exhibited high $p$-values (Figure 5A, Table S1). 
A

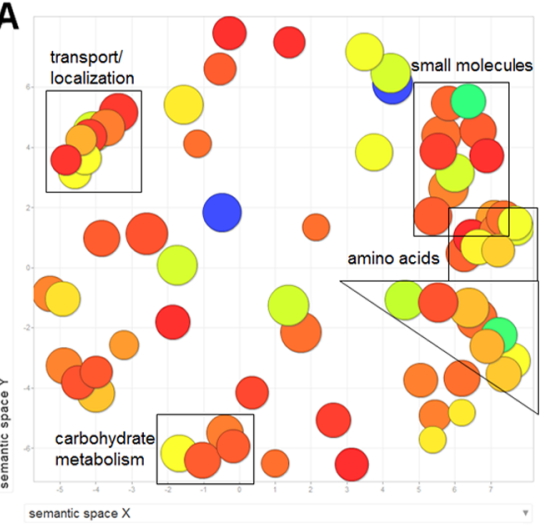

C

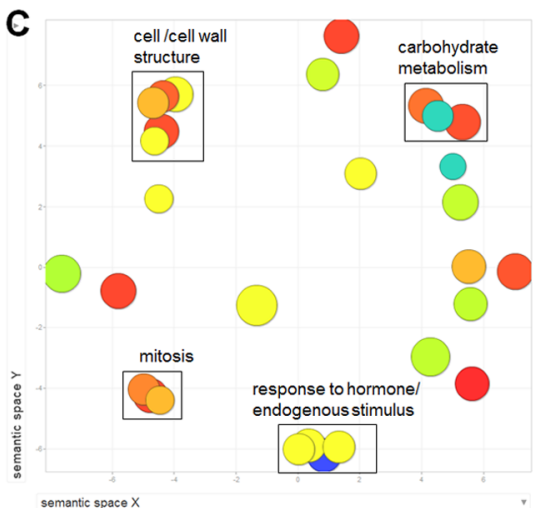

B

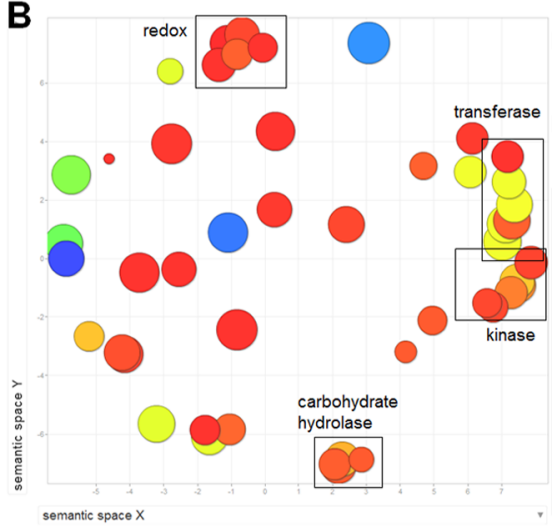

D

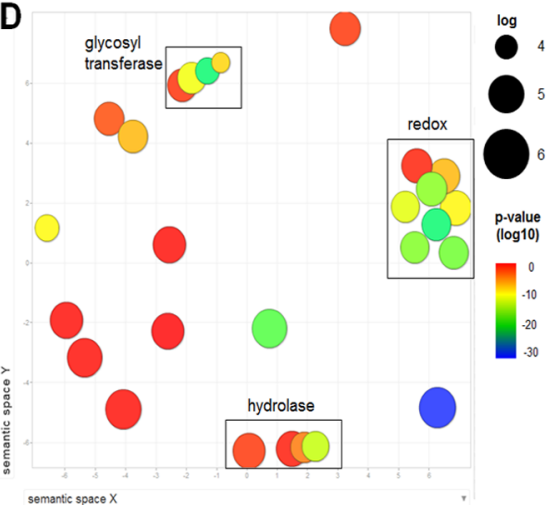

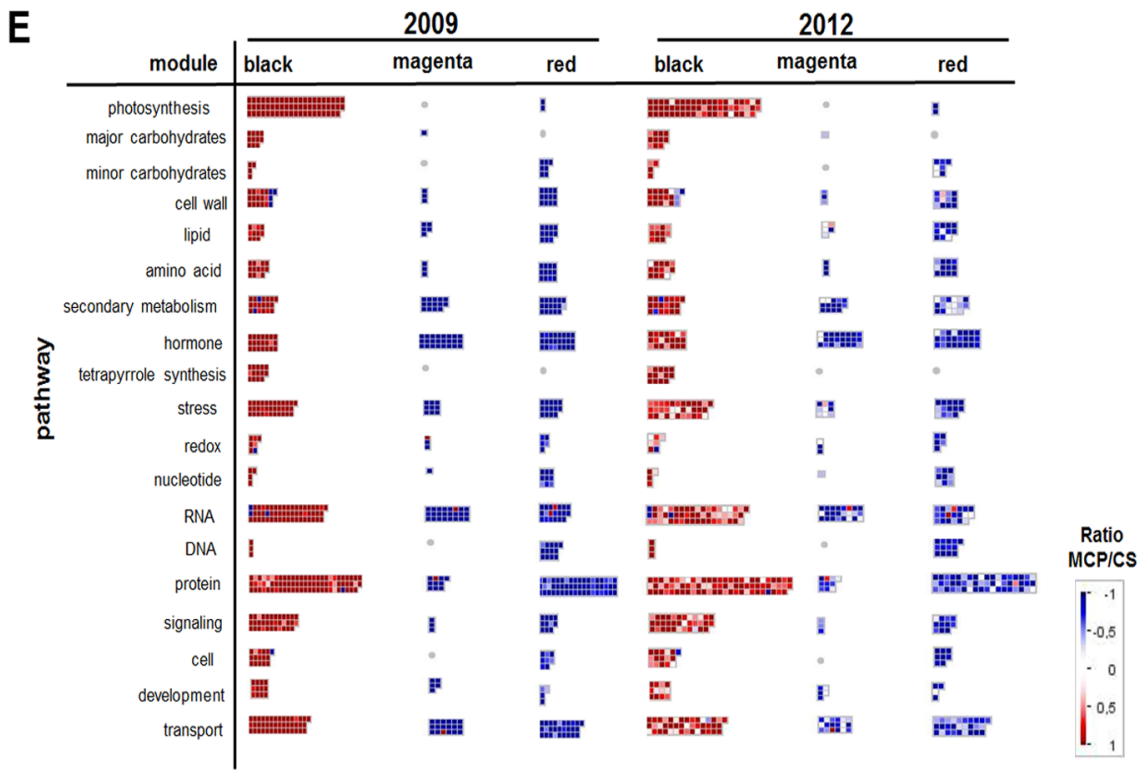

Figure 6. Scatterplot graphs of nonredundant cluster representatives of biological process Gene Ontology (GO) for modules blue (A, B) and brown (C, D), according to REVIGO ${ }^{26}$ Axis coordinates on $x$ and $y$ represent semantic similarity in the two-dimensional space. Metabolism classification according to the Mapman ${ }^{24}$ scheme for modules black, magenta, and red (E). Heatmap represents transcription ration between MCP and CS.

Correlation plots between gene significance (GS) and module membership (MM), with their respective $p$-values, for the modules with weaker association to firmness, soluble solids contents, and total acidity are shown in Figure S4 and Table S1. The relationships between the Eigengene in each module and the sensorial traits were represented as dendrograms, along with the correlation analyses of the trait and the Eigengene summarizing the module (Figure S3).

Intramodular analyses of GS and MM confirmed the validity of module-trait associations with high correlations and low $p$ - value (Figure 5B,C,D). Thus, genes in module blue are associated with the control of fruit soluble solids accumulation and acidity, those in module brown with firmness and acidity, the genes in module black with all investigated quality traits, and those in red with soluble solid contents and firmness (Figure 5).

Functional Analyses of Module Blue. More than $77 \%$ of the DEGs displayed two distinct co-regulation patterns and were classified to modules blue $(48.8 \%, 2960)$ and brown $(29.0 \%, 1766)$ (Figure $4 \mathrm{~A})$. We have used two nonredundant 


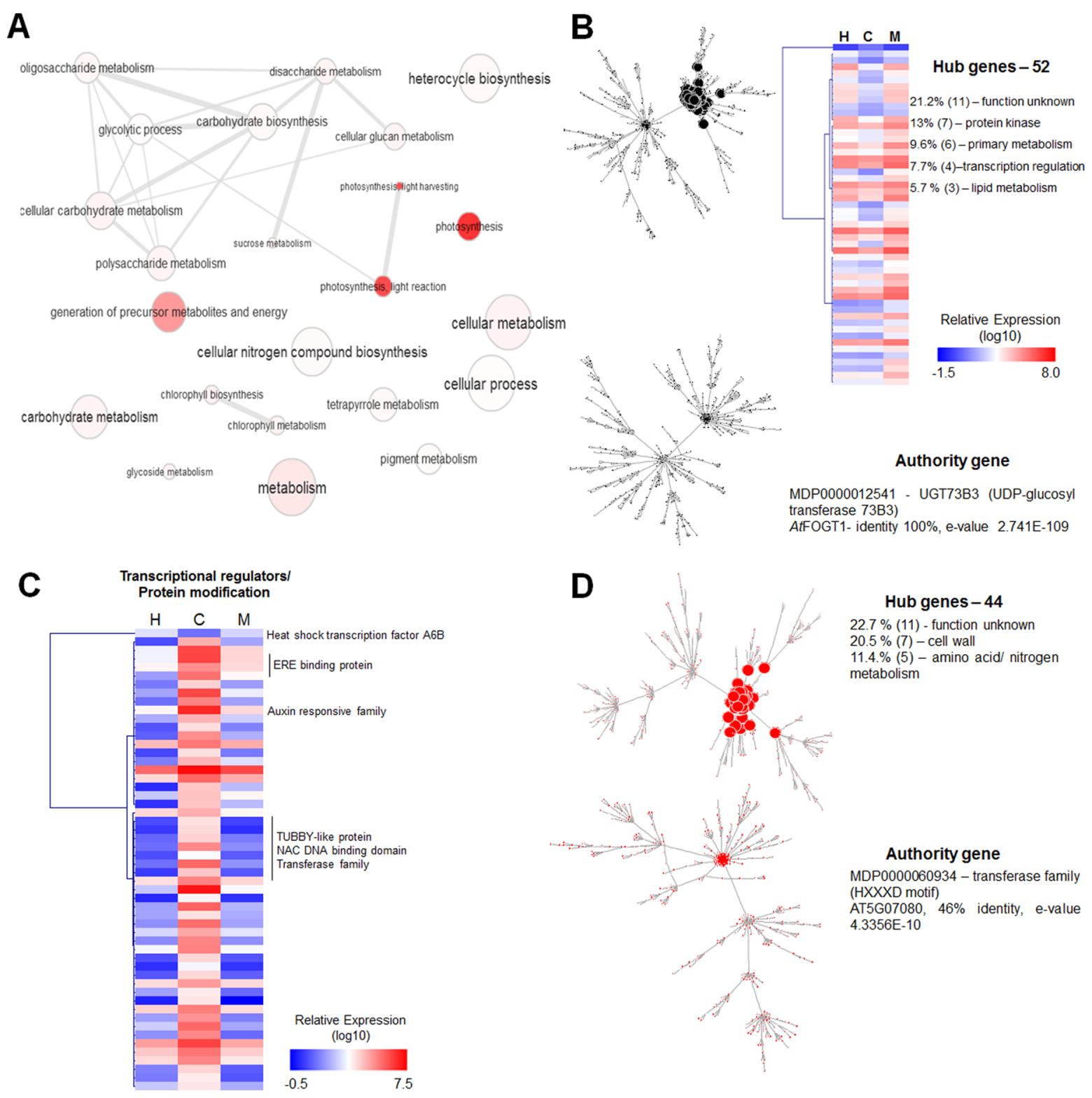

Figure 7. Biological process GO enrichment analyses of co-regulated genes in module black as REVIGO ${ }^{26}$ network (A). Weighted correlation networks for the genes in module black designed according to the Barabasi-Albert preferential attachment model, with hub and authority (B) scores represented by marker size. Hierarchical clustering of the transcriptional profile of genes involved in transcriptional regulation and protein modification in module red $(\mathrm{C})$ at harvest $(\mathrm{H})$ and after 60 days of cold storage alone $(\mathrm{C})$ and with 1-MCP application (M). Weighted correlation networks for the genes in module red designed according to the Barabasi-Albert preferential attachment model, with hub and authority (D) scores represented by marker size.

ontology analysis tools agriGO ${ }^{25}$ and $\mathrm{REVIGO}^{26}$ (Figure 6, Table S2). The ontology classification of the genes to Biological Process showed significant enrichment in metabolic pathways associated with ripening, small molecule metabolic processes, lipid, localization and transport, and inositol catabolism (Figure 6A, Table S2). Molecular function also revealed enrichment in catalytic, hydrolase, and oxidoreductase activity (Figure 6B, Table S2). Significant cellular localization ontology was also found for membranes (Table S2).

Functional Analyses of Module Brown. Gene ontology analyses of module brown reveled enrichment in a smaller number of terms of biological processes (32), cellular component (5), and molecular function (17) (Figure 6C,D, Table S2). The module was significantly enriched in genes associated with the response to biotic stimulus, carbohydrate metabolism, cellular structure, and cell organization and response to hormone and endogenous stimulus (Figure 6C). The most frequent cellular component ontology terms in module brown were plasma membrane and the DNA clamp acting as a processivity factor for DNA polymerase $\delta$, essential for replication, the proliferating cell nuclear antigen (PCNA) complex (Table S2).

Functional Analyses of Modules Black, Red, and Magenta. The predominant factor on the regulation of genes in module black was transcriptional induction by 1-MCP, with a stronger effect of the hormone inhibitor in 2009 (Figure $4 \mathrm{D}$, Figure 6E). Ontology enrichment analyses demonstrated high frequency of genes associated with photosynthesis, generation of precursor metabolites, carbohydrate metabolic processes, and cellulose metabolic processes (Figure 6E, Figure 


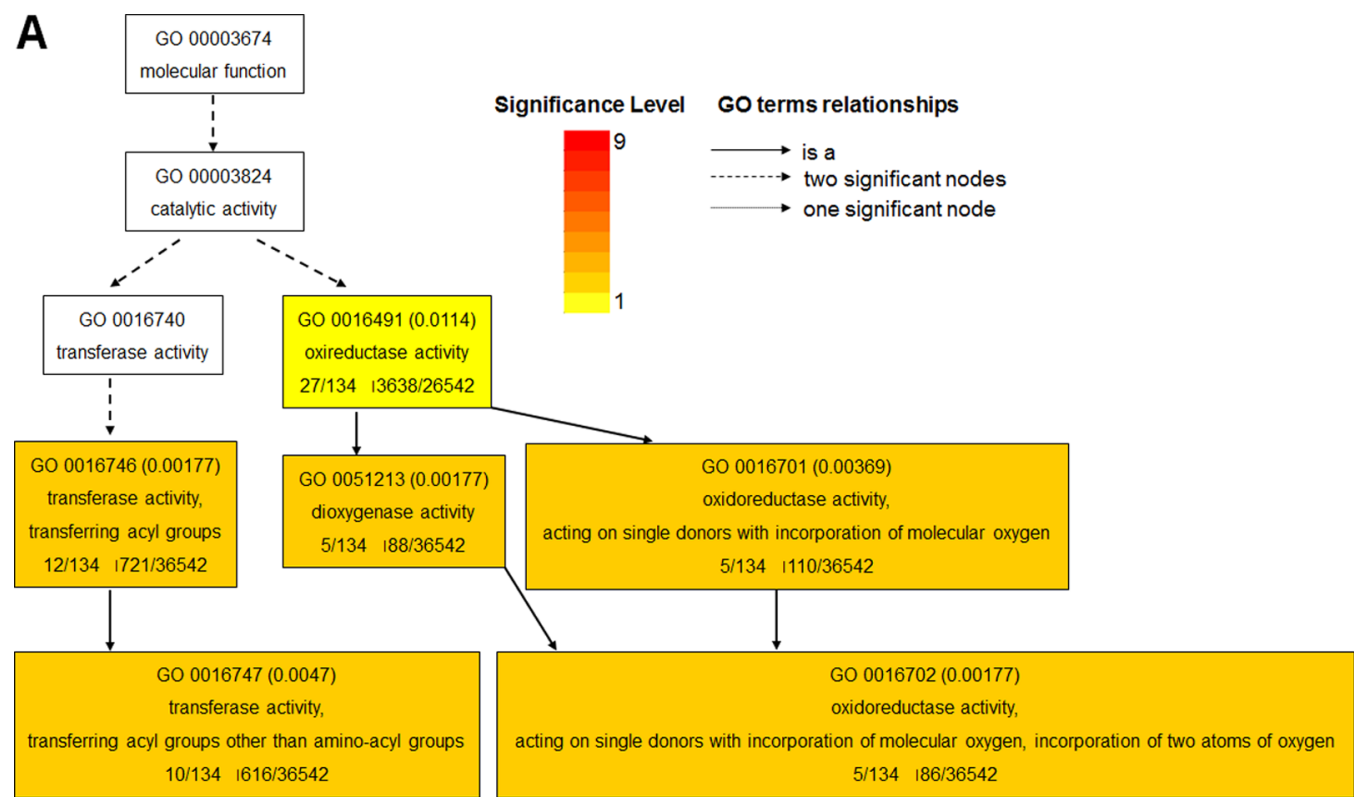

B

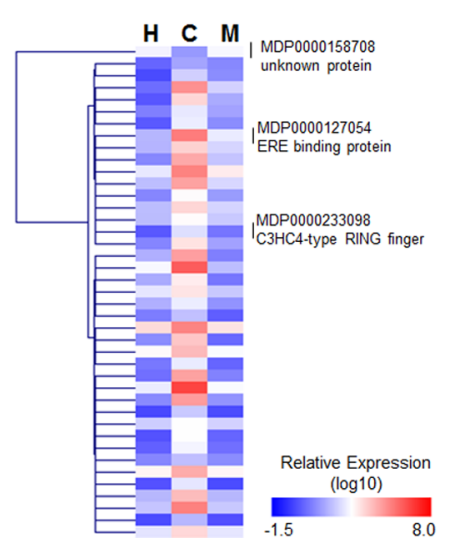

C

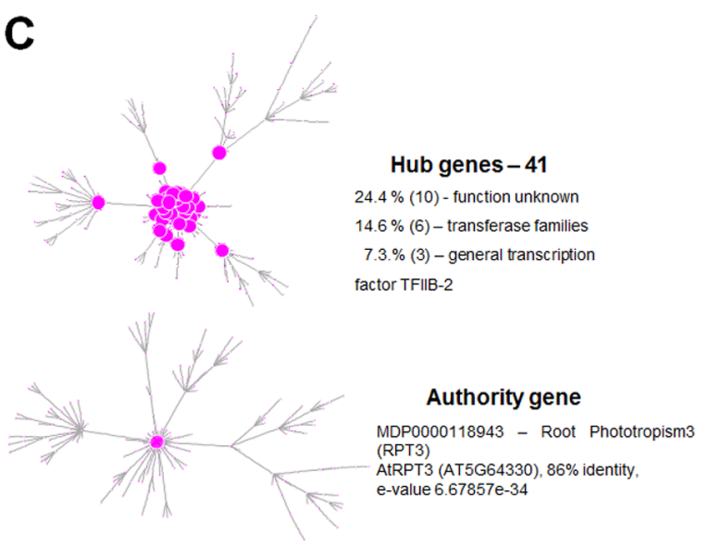

Figure 8. Molecular function GO enrichment analyses of co-regulated genes in module magenta, represented as AgriGO ${ }^{25}$ chart $(\mathrm{A})$. Hierarchical clustering of the transcriptional profile of hub genes in module magenta at harvest $(\mathrm{H})$ and after 60 days of cold storage alone $(\mathrm{C})$ and with 1-MCP application (M) (B). Weighted correlation networks for the genes in module magenta designed according to the Barabasi-Albert preferential attachment model with hub and authority $(\mathrm{C})$ scores represented by marker size.

7 A, Table S2). Further analyses of the connection strength in the adjacency matrix of module black network revealed the presence of 52 hub genes, most of them with unknown function $(21.2 \%, 11)$ (Figure $7 \mathrm{~B})$. The authority gene in the module corresponded to MDP0000012541, a UDP-glucosyl transferase 73B3 (Figure 7B).

Module red represents approximately $6.6 \%$ of the DEGs and consists of genes whose transcription is regulated mainly by environmental differences in harvesting year (Figure 4D, Figure $6 \mathrm{E})$. Significant enrichment in gene ontology terms in module red were associated with oxidation and reduction and negative regulation of biological processes (Table S2). The enrichment in ontology terms associated with negative regulation prompted the investigation of the expression profile of genes with predicted functions in hormone response and protein modification (Figure 7C). Two genes coding for ethylene responsive element binding proteins (MDP0000249060 and MDP0000262020) in the module were induced by CS and repressed by MCP, although not reaching levels as low as those observed at $\mathrm{H}$ (Figure $7 \mathrm{C}$ ). Similar regulation patterns were observed for other sequences associated with hormone responsive transcription factors (Figure $7 \mathrm{C}$ ). In the module,
44 (11\%) genes exhibited the highest hub scores, with the majority consisting of sequences with unknown function $(22.7 \%, 11)$ (Figure 7D). Authority gene in the module red corresponded to MDP0000060934, a gene containing a HXXXD motif, classified to the transferase family (Figure 7D).

Low temperatures during storage was the main factor controlling the expression of genes in module magenta (Figure $4 \mathrm{~F}$, Figure $6 \mathrm{E}$ ). The module was enriched in biological process ontology associated with transcriptional regulation as biological process, and to several terms derived from catalytic activity in molecular function (Figure 8A, Table S2). The transcription of most genes in module magenta was induced by CS, except for MDP0000158708, a transcript with no significant homology to functionally characterized sequences, which was repressed by $\mathrm{CS}$, returning to $\mathrm{H}$ levels under MCP treatment (Figure 8B). Forty-one genes in the module had high hub scores (19.6\%), with most of them $(24.4 \%, 10)$ of unknown function (Figure $8 \mathrm{C})$. The authority gene in the module corresponds to MDP0000118943, a BTB (Broad-complex, Tramtrack and Bric-a-brac)/POZ domain (Poxvirus and Zinc finger) domain containing protein, similar to Arabidopsis ROOT PHOTOTROPISM3 (Figure 8C). 


\section{DISCUSSION}

Quality Attributes Are Partially Retained by Postharvest Techniques and Affected Distinctly by the Year. In commercial apple production, cold storage alone or in combination with atmosphere manipulations and ethylene blockage is essential to extending the fruit availability window. Apple flesh firmness is the crucial determinant for consumer acceptance, followed by a desired balance between sweetness and acidity. ${ }^{3,5,6,9}$ However, physicochemical analyses have demonstrated that the postharvest techniques differentially affect these attributes (Figure 1B). After 60 days, CS or MCP treatments did not fully prevent the increase in soluble solids and decrease in acidity, in agreement with the unidirectional character of ripening in contrast to other plastic developmental processes in plants. ${ }^{10-12}$ In contrast, inhibition of ethylene perception by $1-\mathrm{MCP}$ in fruits kept at low temperatures retained flesh firmness to levels similar to those at harvest (Figure 1B). In contrast, the blockage of ethylene perception did not enhance firmness conservation of 'Galaxy' apples stored under dynamic controlled atmosphere monitored by respiratory quotient. ${ }^{30}$ Interestingly, the authors also report the lack of influence of the maturity state at harvest on conservation. ${ }^{30}$

In commercial apple production, the optimum harvest window is determined by variable number of days of acceptable maturity that is determined by the genomic context of the cultivar, environmental conditions, and desired period of storage. $^{31}$ Recently, large scale chemical profiling and physicochemical analyses have demonstrated that the main factors affecting fruit quality traits, including soluble solids content and titratable acidity, are the cultivar and the year of cropping, with no significant effects of the orchard management system. ${ }^{32}$ These observations agree with the marked effect of the year on the contents of soluble solids, acidity, and genome wide transcriptional profiling at harvest. Moreover, the gene expression analyses confirm that significant differential gene activity is retained in the harvest window of acceptable maturity. The weighted network analyses also demonstrated that the influence of the growing season on gene expression was distinct for groups of genes associated with different quality traits: the differences in gene expression between 2009 and 2012 were less significant than those found in fruits of later developmental stage (60 days older) for the genes in module blue and brown, whereas the year differences were significant in fruits submitted to the same postharvest treatment for the genes in modules black, red, and magenta. Thus, the influence of the environmental factors during the year are distinct depending on the transcriptional programs in apples after harvest.

Transcriptional Changes Underlying Apple Quality Traits after Harvest and during Storage. Ripening of fleshy fruit is one of the last developmental phases of fruit ontogeny and involves many genetic, biochemical, and physiological modifications. These changes include the accumulation of pigments and sugars, the production of aromatic compounds, and flesh softening. ${ }^{13}$ Ripening enhances fruit organoleptic properties, making them adequate for human consumption. However, fruit quality diminishes as ripening reaches an advanced stage, mainly due to fruit oversoftening, increased pathogen susceptibility and the development of undesirable flavor and skin color, leading to important economic losses during postharvest fruit management. ${ }^{2,6,9,16}$
Complex carbohydrates, such as cellulose, pectic polysaccharides and xyloglucan, are the major compounds in plant cell walls, along with smaller portions of hemicelluloses, as arabinoxylans or glucomannans. ${ }^{33}$ The development, ripening and senescence of apples are associated with modifications in the cell wall polysaccharides, including depolymerization, dissolution, and spatial rearrangement of pectins, hemicellulose, and cellulose. ${ }^{13,34}$ Previous studies have associated the inhibition of ethylene-mediated signaling to the transcriptional repression of genes coding for cell wall hydrolyzing enzymes in fruits. ${ }^{2,5-9}$ Our results also demonstrate the transcriptional repression of a portion of genes associated with carbohydrate hydrolysis in response to CS and MCP, such as a subset of polygalacturonase, pectin methylsterase, xylosidase, and glycosyl hydrolases in general. However, a previously unreported induction in the transcription of genes involved in the sucrosesucrose cycle (futile cycle) ${ }^{35}$ was also observed in response to ethylene perception blockage. In Royal Gala apples, SUCROSE SYNTHASE (SUS) expression decreased in ripe fruits where starch degradation is prevalent. ${ }^{36}$ The transcription of SUS was increased by ethylene blockage in our study, indicating that in the absence of the hormone, the fruit resumes a set of metabolic activities that were suppressed during ripening in the presence of ethylene. Indirectly, the accumulation of glucose and sucrose, associated with the reduced respiration rates observed under MCP would lead to higher levels of carbon backbone for the biosynthesis of novel carbohydrates. In fruits after harvest, the function of SUS is reversed to cleaving sucrose to form fructose and UDP-glucose (UDPG), in contrast to its traditional role in synthesizing sucrose from UDPG. ${ }^{37}$ Fructose is then phosphorylated to fructose 6-phosphate, which can be used in glycolysis, tricarboxylic acid cycle, or combined with UDPG in sucrose resynthesis via sucrose phosphate synthase. ${ }^{38}$ Although the breakdown and resynthesis of sucrose may seem like a wasteful process, it is associated with a more precise control of carbohydrate partitioning in maize endosperm, since the metabolic stability of sucrose facilitates its symplastic transport to tissues where starch biosynthesis occurs. ${ }^{35}$ Moreover, the increased activity of sucrose-phosphate synthase activates starch production, possibly due to an increase in the sucrose to hexose ratio. High levels of sucrose may also function as cellular protectants to osmotic and cold stress. ${ }^{39}$

A similar reappraisal of metabolic pathways associated with more juvenile stages of fruit development in response to 1MCP was observed for genes encoding proteins associated with photosynthesis. During ripening, photosynthetically active chloroplasts undergo a physiological transition to chromoplasts, colored plastids containing lipid-soluble pigments. ${ }^{40} \mathrm{~A}$ marked transcriptional and translational repression of genes related to photosynthesis accompanies the ripening transition to chromoplasts, possibly via a plastid-specific repression mechanism. ${ }^{40}$ The role of ethylene on photosynthesis in vegetative structures appears to be the reverse, since increased ethylene production in response to salt and heavy metal stresses induced photosynthesis. ${ }^{41,42}$

The inhibition of ethylene signaling also alleviated the repression of the transcription of a subset of genes associated with maintenance of the cellular redox status, such as the conserved redox proteins glutathione/glutaredoxin (GRX) and thioredoxin (TRX) systems.

The regulation of thiol-disulfide redox status has a prevalent role in plant defense mechanisms by preserving cellular redox homeostasis and structural and regulatory functions for many 
proteins. ${ }^{43}$ In vegetative Arabidopsis organs, genetic studies have demonstrated that ethylene mediated signaling induces the transcription of redox proteins in response to different abiotic stresses. ${ }^{44,45}$ Thus, taken together our results demonstrate that in apple, where ethylene has a prevalent role in controlling ripening, its suppression by application of the competitive inhibition allows derepression of the transcription of photosynthesis genes and of a subset of redox proteins.

Differentially Expressed Genes Exhibit Five Distinct Regulatory Patterns. The expression patterns of the significant DEGs were classified in five distinct transcriptional programs, or co-regulated gene modules, using weighted gene correlation network analysis (WGCNA). ${ }^{23}$ The characterization of the functional independence of biological parts and devices or their modularity is essential to the design and modeling steps of plant synthetic biology. ${ }^{46}$ Conserved modules are often recombined and rearranged in nature to create novel functions, depending on their context dependency. ${ }^{23,46}$ The identification of transcriptionally co-regulated modules helped the characterization of genes associated with fruit acidity and anthocyanin production in diverse genomic contexts in apple and the identification of genes and metabolites associated with senescence and biotic stress resistance in other species. ${ }^{47,48}$

The identified co-regulated modules correspond to distinct transcriptional programs responding differently to the tested conditions $(\mathrm{H}, \mathrm{CS}$, and $\mathrm{MCP})$ but also revealing the effects of the environmental conditions during the two investigated growing seasons. The differential weight of the associations between the transcriptional programs and the quality traits also provided further insight on their regulation in response to postharvest techniques. The largest transcriptional program corresponds to genes preferentially controlled by development and is positively associated with acidity and accumulation of soluble solids. The second largest program is negatively associated with firmness and positively with fruit acidity and consists of genes exhibiting differential response to the harvesting seasons. Thus, distinct transcriptional programs with differential responses to development, conservation techniques, and growing season differences control quality traits in apples after harvest.

Transcriptional Programs Affecting Flesh Firmness. Firmness is considered one of the most important traits for consumer acceptance in apples. ${ }^{1-3}$ It is a complex phenotype and is thought to be controlled by several genome regions and their interactions with the environment. ${ }^{2,4-6}$ In our uniform genomic context ('Gala'), three distinct transcriptional modules were demonstrated to exert opposite effects on flesh firmness, genes in modules red and brown negatively and those in black positively associated with the trait.

Previous studies have associated the maintenance of fruit firmness to impaired transcription or activity of cell wall carbohydrate hydrolyzing enzymes or to defective ethylene synthesis or signaling. ${ }^{2,4,5,9}$ The results of our weighted correlation analyses of gene expression demonstrate that a two-time mechanism controls firmness in apples after harvest: the first component consists of transcriptional programs preferentially regulated by development that negatively affect trait, whereas the second component consists of a transcriptional program regulated by postharvest techniques CS and MCP. Interestingly, the program exhibiting a marked response to the blockage of ethylene perception resumes the transcription of several genes associated with anabolic pathways, suppressed in the later stages of fruit development. These observations agree with recent reports that the inhibition of ethylene perception by 1-MCP derepressed the transcription of genes related to auxin signaling and metabolism, transcription factors, and photosynthesis in apples after harvest. ${ }^{8}$ Our results further demonstrate that the transcriptional reappraisal of vegetative and anabolic pathways in response to blockage of ethylene perception remains enforced even after 60 days of storage and is associated with firmness control.

Transcriptional Programs Affecting the Contents of Soluble Solids. Genetic studies employing segregating populations demonstrated the involvement of several genomic regions in the control of the contents of soluble solids in apples. ${ }^{49,50}$ Soluble sugars in apples (peel and flesh) consist mainly of fructose, sucrose, arabinose, galactose, and galacturonic acid as the main component monosaccharides. Two genomic regions in Linkage Group 01, separated by $145 \mathrm{~kb}$ in the apple reference genome, were associated with fructose and sucrose contents in 'Jonathan' and 'Golden Delicious', respectively. ${ }^{49}$ Thus, the contents of chemically distinct soluble sugars are likely to depend on different genomic regions in apple. In 'Gala', gene modules blue and red were positively correlated to the contents of soluble solids. The expression summary of these programs demonstrates a significant effect of developmental time and the growing season in gene regulation, in agreement with observations that the year exerts a predominant influence on sugar contents in apple, hierarchically superior to orchard management systems. ${ }^{32}$ Moreover, the ineffectiveness of postharvest techniques in fully preventing the increase in the contents of soluble sugars also agrees with the negative correlation observed for the trait and the gene module preferentially regulated by development and enriched in catabolic pathway ontologies (blue).

Further functional analyses of the co-regulated modules positively associated with the contents of soluble sugars revealed that the genes with higher number of connections in programs red and black are associated with transcriptional regulation, protein-protein interaction and modification, and chromatin remodeling. The authority genes in the modules correspond to transferases: a UDP-glucosyltransferase (UGT) and a HXXXD-motif transferase. In higher plants, UGTs belong to a superfamily consisting of 56 protein families, functionally involved in the biosynthesis of specialized metabolites, such as flavonoids, phenylpropanoids, terpenoids, and steroids, and the regulation of plant hormones. ${ }^{51}$ Acyltransferases containing the HXXXD motif, also called BAHD family of acyltransferases, were named based on the four biochemically characterized enzymes of the family (BEAT, AHCT, HCBT, and DAT), and are also associated with the biosynthesis of specialized metabolites, such as cuticle lipids, volatile esters, modified anthocyanins, defense compounds, and phytoalexins. ${ }^{51}$ In Arabidopsis, UGTs were demonstrated to control abscisic acid (ABA) homeostasis and ABA-mediated seed germination. ${ }^{51}$ Among the co-regulated transcripts, a significant portion is predicted to function in transcriptional regulation, protein-protein interactions, and protein modifications, along with a smaller number similar to 9-cisepoxycarotenoid dioxygenase (NCED), the key enzyme in $\mathrm{ABA}$ biosynthesis, and proteins induced by biotic stress, suggesting a role for other hormone-mediated signaling components in the developmental responses of mature, detached fruits in apple, as observed in peach. ${ }^{52}$ In fruits of Myrica pensylvanica, bayberry, a HXXXD-containing acyltransferase was demonstrated to control a novel biosynthetic 
pathway of cutin triacylglycerols. ${ }^{53}$ The authority role of a similar acyltransferase may indicate a similar function in stored apples.

Transcriptional Programs Affecting Fruit Acidity. Several genomic regions control fruit acidity in apple and are differentially associated with the chemicals responsible for the trait. ${ }^{49}$ Moreover, the trait depends on a wide range of cellular processes, such as organic acid biosynthesis, vacuole sequestration, and degradation. ${ }^{49,54}$ The complexity of the regulatory levels responsible for acidity in fleshy fruits agrees with the observation that distinct transcriptional modules correlate with the trait. Module blue was simultaneously associated with soluble solid contents and acidity, although with opposite effects, whereas black had the same effect on the traits. The chemical characterization of soluble solids and acid components in cultivated and wild apple genotypes also suggests a complex association between acidity and sugar composition. ${ }^{54}$ The demonstration that distinct transcriptional modules are associated with the contents of soluble solids and fruit acidity in apple suggests that, at least partially, the interaction occurs at the transcriptional level. Moreover, the positive association of transcriptional programs mainly controlled by developmental time (blue and brown) to acidity is in agreement with the observation that postharvest technologies do not fully prevent the loss of organic acids in the later stages of apple ripening.

Our results demonstrate that conservation techniques do not fully prevent ripening processes in apple, although inhibition of ethylene perception effectively reduces firmness loss. Contrasting transcriptional programs, identified by weighted network analyses, underlie postharvest development and are correlated to quality traits. The inhibition of ethylene perception and signaling leads to a sustained upregulation of genes in anabolic and vegetative development pathways in the fruits for up to 60 days after harvest. Complex phenotype traits, critical for consumer appreciation, were associated with subsets of differentially regulated genes.

\section{ASSOCIATED CONTENT}

\section{S Supporting Information}

The Supporting Information is available free of charge on the ACS Publications website at DOI: 10.1021/acs.jafc.7b01425.

Primer information for RT-qPCR expression validation (Table S3), GO classification of the DEGs in biological process, cellular component, and molecular function (Figure S1), REVIGO scatterplot of GO classification of the DEGs (Figure S2), correlation between the modules eigengenes (ME) and the investigated quality traits (Figure S3), gene significance and module membership for the modules with non-significant association to the quality traits (Figure S4), heatmap representation of the gene network in co-regulated modules (Figure S5), and validation of microarray gene expression profiles (Figure S6) (PDF)

Table S1, Gene Significance (GS) and Module Membership (MM) for the quality traits (XLSX)

Gene ontology (GO) classification of the DEGs (XLSX)

Table S4, hub and authority scores (XLSX)

\section{Accession Codes}

Microarray data is available at Gene Expression Omnibus (http://www.ncbi.nlm.nih.gov/geo/) under the accession number GSE59728.

\section{AUTHOR INFORMATION}

\section{Corresponding Author}

*César Luis Girardi. E-mail: cesar.girardi@embrapa.br. Phone: +55 (54) 3455-8000. Fax: +55 (54) 3455-2176.

ORCID

César Luis Girardi: 0000-0003-1888-8872

\section{Present Address}

${ }^{\perp}$ Universidade Tecnológica Federal do Paraná, Pato Branco, PR 85503-390, Brazil.

\section{Author Contributions}

T.T.S. participated in the design, performed physiological and gene expression analyses, performed statistical data analyses, and drafted the manuscript. T.F., M.B., M.O.-B. and J.-P.R. participated in gene expression and statistical data analyses. V.Q. contributed to data analyses, results interpretation, and manuscript drafting. C.V.R. and C.L.G. designed the experiments and drafted the manuscript. All authors have read and approved the final manuscript.

\section{Funding}

The work was supported by the Conselho Nacional de Desenvolvimento Científico e Tecnológico (CNPq, 306771/ 2014-4 and 441856/2014-4), Empresa Brasileira de Pesquisa Agropecuária (Embrapa, 02.13.05.014.00.00), Coordenação de Aperfeiçoamento de Pessoal de Nivel Superior (CAPES/ Embrapa, 15/2014), and Comité Français d'Évaluation de la Coopération Universitaire et Scientifique avec le Brésil (CapesCofecub, 631/09) through scholarship and financial support.

\section{Notes}

The authors declare no competing financial interest.

\section{ACKNOWLEDGMENTS}

Authors thank Mr. Nelson Balardin for providing access and authorizing apple sample collections from his commercial orchard.

\section{ABBREVIATIONS USED}

$\mathrm{H}$, harvest; CS, cold storage; MCP, 1-methylcyclopropene; FF, flesh firmness; TA, titratable acidity; SSC, soluble solids contents; RT, room temperature; Cy3, cyanine-3; Cy5, cyanine-5; BH, Benjamini-Hochberg; GDR, Genome Database for Rosaceae; GEO, Gene Expression Omnibus; GO, gene ontology; FDR, false discovery rate; RT-qPCR, reverse transcription quantitative polymerase chain reaction; $C D$, coding sequence; DEGs, differentially expressed genes; WGCNA, weighted gene correlation network analysis; EC, Enzyme Commission number; GS, gene significance; MM, module membership; TOM, topological overlap matrix; HCA, hierarchical clustering analysis

\section{REFERENCES}

(1) Costa, F.; Cappellin, L.; Fontanari, M.; Longhi, S.; Guerra, W.; Magnago, P.; Gasperi, F.; Biasioli, F. Texture dynamics during postharvest cold storage ripening in apple (Malus $\times$ domestica Borkh.). Postharvest Biol. Technol. 2012, 69, 54-63.

(2) Storch, T. T.; Finatto, T.; Pegoraro, C.; Dal Cero, J.; Laurens, F.; Rombaldi, C. V.; Quecini, V.; Girardi, C. L. Ethylene-dependent regulation of an $\alpha$-l-arabinofuranosidase is associated to firmness loss in "Gala" apples under long term cold storage. Food Chem. 2015, 182, $111-119$.

(3) Bonany, J.; Buehler, A.; Carbó, J.; Codarin, S.; Donati, F.; Echeverria, G.; Egger, S.; Guerra, W.; Hilaire, C.; Höller, I.; Iglesias, I.; Jesionkowska, K.; Konopacka, D.; Kruczyńska, D.; Martinelli, A.; 
Pitiot, C.; Sansavini, S.; Stehr, R.; Schoorl, F. Consumer eating quality acceptance of new apple varieties in different European countries. Food Qual. Pref. 2013, 30, 250-259.

(4) Migicovsky, Z.; Gardner, K. M.; Money, D.; Sawler, J.; Bloom, J. S.; Moffett, P.; Chao, C. T.; Schwaninger, H.; Fazio, G.; Zhong, G. Y.; Myles, S. Genome to phenome mapping in apple using historical data. Plant Genome 2016, 9, 2.

(5) Bink, M. C.; Jansen, J.; Madduri, M.; Voorrips, R. E.; Durel, C. E.; Kouassi, A. B.; Laurens, F.; Mathis, F.; Gessler, C.; Gobbin, D.; Rezzonico, F.; Patocchi, A.; Kellerhals, M.; Boudichevskaia, A.; Dunemann, F.; Peil, A.; Nowicka, A.; Lata, B.; Stankiewicz-Kosyl, M.; Jeziorek, K.; Pitera, E.; Soska, A.; Tomala, K.; Evans, K. M.; Fernández-Fernández, F.; Guerra, W.; Korbin, M.; Keller, S.; Lewandowski, M.; Plocharski, W.; Rutkowski, K.; Zurawicz, E.; Costa, F.; Sansavini, S.; Tartarini, S.; Komjanc, M.; Mott, D.; Antofie, A.; Lateur, M.; Rondia, A.; Gianfranceschi, L.; van de Weg, W. E. Bayesian QTL analyses using pedigreed families of an outcrossing species, with application to fruit firmness in apple. Theor. Appl. Genet. 2014, 127, 1073-1090.

(6) Nobile, P. M.; Wattebled, F.; Quecini, V.; Girardi, C. L.; Lormeau, M.; Laurens, F. Identification of a novel $\alpha$-L-arabinofuranosidase gene associated with mealiness in apple. J. Exp. Bot. 2011, 62, 4309-4321.

(7) Zhang, Z.; Wang, N.; Jiang, S.; Xu, H.; Wang, Y.; Wang, C.; Li, M.; Liu, J.; Qu, C.; Liu, W.; Wu, S.; Chen, X.; Chen, X. Analysis of the xyloglucan endotransglucosylase/hydrolase gene family during apple fruit ripening and softening. J. Agric. Food Chem. 2017, 65 (2), 429434.

(8) Tadiello, A.; Longhi, S.; Moretto, M.; Ferrarini, A.; Tononi, P.; Farneti, B.; Busatto, N.; Vrhovsek, U.; Molin, A. D.; Avanzato, C.; Biasioli, F.; Cappellin, L.; Scholz, M.; Velasco, R.; Trainotti, L.; Delledonne, M.; Costa, F. Interference with ethylene perception at receptor level sheds light on auxin and transcriptional circuits associated with the climacteric ripening of apple fruit (Malus $x$ domestica Borkh.). Plant J. 2016, 88 (6), 963-975.

(9) Tacken, E.; Ireland, H.; Gunaseelan, K.; Karunairetnam, S.; Wang, D.; Schultz, K.; Bowen, J.; Atkinson, R. G.; Johnston, J. W.; Putterill, J.; Hellens, R. P.; Schaffer, R. J. The role of ethylene and cold temperature in the regulation of the apple POLYGALACTURONASE1 gene and fruit softening. Plant Physiol. 2010, 153 (1), 294-305.

(10) Galli, M.; Gallavotti, A. Expanding the regulatory network for meristem size in plants. Trends Genet. 2016, 32 (6), 372-383.

(11) Sawicki, M.; Ait Barka, E.; Clément, C.; Vaillant-Gaveau, N.; Jacquard, C. Cross-talk between environmental stresses and plant metabolism during reproductive organ abscission. J. Exp. Bot. 2015, 66 (7), 1707-1719.

(12) Van de Poel, B.; Smet, D.; Van Der Straeten, D. Ethylene and hormonal cross talk in vegetative growth and development. Plant Physiol. 2015, 169 (1), 61-72.

(13) Johnston, J. W.; Gunaseelan, K.; Pidakala, P.; Wang, M.; Schaffer, R. J. Co-ordination of early and late ripening events in apples is regulated through differential sensitivities to ethylene. J. Exp. Bot. 2009, 60, 2689-2699.

(14) Li, D. P.; Xu, Y. F.; Sun, L. P.; Liu, L. X.; Hu, X. L.; Li, D. Q.; Shu, H. R. Salicylic acid, ethephon, and methyl jasmonate enhance ester regeneration in 1-MCP-treated apple fruit after long-term cold storage. J. Agric. Food Chem. 2006, 54 (11), 3887-3895.

(15) Watkins, C. B. The use of 1-methylcyclopropene (1-MCP) on fruits and vegetables. Biotechnol. Adv. 2006, 24, 389-409.

(16) Leisso, R. S.; Gapper, N. E.; Mattheis, J. P.; Sullivan, N. L.; Watkins, C. B.; Giovannoni, J. J.; Schaffer, R. J.; Johnston, J. W.; Hanrahan, I.; Hertog, M. L.; Nicolai, B. M.; Rudell, D. R. Gene expression and metabolism preceding soft scald, a chilling injury of 'Honeycrisp' apple fruit. BMC Genomics 2016, 17, 798.

(17) de Mendiburu, F. Package 'Agricolae'. 2016. https://cran.rproject.org/web/packages/agricolae/agricolae.pdf. Accessed in 10/16/ 2016.
(18) Zeng, Y.; Yang, T. RNA isolation from highly viscous samples rich in polyphenols and polysaccharides. Plant Mol Biol Rep. 2002, 20, $417 \mathrm{a}-417 \mathrm{e}$

(19) Storch, T. T.; Pegoraro, C.; Finatto, T.; Quecini, V.; Rombaldi, C. V.; Girardi, C. L. Identification of a novel reference gene for apple transcriptional profiling under postharvest conditions. PLoS One 2015, 10 (3), e0120599.

(20) Celton, J.-M.; Gaillard, S.; Bruneau, M.; Pelletier, S.; Aubourg, S.; Martin-Magniette, M.-L.; Navarro, L.; Laurens, F.; Renou, J.-P. Widespread anti-sense transcription in apple is correlated with siRNA production and indicates a large potential for transcriptional and/or post-transcriptional control. New Phytol. 2014, 203, 287-299.

(21) R Development Core Team. R: A language and environment for statistical computing. 2014. R Foundation for Statistical Computing, Vienna, Austria. ISBN 3-900051-07-0, http://www.R-project.org. Accessed in 10/15/2016.

(22) Ritchie, M. E.; Phipson, B.; Wu, D.; Hu, Y.; Law, C. W.; Shi, W.; Smyth, G. K. limma powers differential expression analyses for RNAsequencing and microarray studies. Nucleic Acids Res. 2015, 43 (7), e47.

(23) Langfelder, P.; Horvath, S. WGCNA: an R package for weighted correlation network analysis. BMC Bioinf. 2008, 9, 559.

(24) Thimm, O.; Bläsing, O.; Gibon, Y.; Nagel, A.; Meyer, S.; Krüger, P.; Selbig, J.; Müller, L. A.; Rhee, S. Y.; Stitt, M. MAPMAN: a userdriven tool to display genomics data sets onto diagrams of metabolic pathways and other biological processes. Plant J. 2004, 37 (6), 914939.

(25) Du, Z.; Zhou, X.; Ling, Y.; Zhang, Z.; Su, Z. agriGO: a GO analysis toolkit for the agricultural community. Nucleic Acids Res. 2010, 38, W64-W70.

(26) Supek, F.; Bošnjak, M.; Škunca, N.; Šmuc, T. REVIGO summarizes and visualizes long lists of gene ontology terms. PLoS One 2011, 6 (7), e21800.

(27) Nishida, K.; Ono, K.; Kanaya, S.; Takahashi, K. KEGGscape: a Cytoscape app for pathway data integration. F1000Research 2014, 3, 144.

(28) Csardi, G.; Nepusz, T. The igraph software package for complex network research, Int. J. Complex Systems 2006, 1695. http://igraph.sf. net. Accessed in 01/20/2017.

(29) Untergasser, A.; Nijveen, H.; Rao, X.; Bisseling, T.; Geurts, R.; Leunissen, J. A. Primer3Plus, an enhanced web interface to Primer3. Nucleic Acids Res. 2007, 35, W71.

(30) Thewes, F. R.; Brackmann, A.; Anese, R. O.; Ludwig, V.; Schultz, E. E.; Dos Santos, L. F.; Wendt, L. M. Effect of dynamic controlled atmosphere monitored by respiratory quotient and 1methylcyclopropene on the metabolism and quality of 'Galaxy' apple harvested at three maturity stages. Food Chem. 2017, 222, 84-93.

(31) Kupferman, E. Maturity and storage of Gala, Fuji, and Braeburn apples. Tree Fruit Postharvest J. 1994, 5 (3), 10-15.

(32) Le Bourvellec, C.; Bureau, S.; Renard, C. M.; Plenet, D.; Gautier, H.; Touloumet, L.; Girard, T.; Simon, S. Cultivar and year rather than agricultural practices affect primary and secondary metabolites in apple fruit. PLoS One 2015, 10 (11), e0141916.

(33) Cosgrove, D. J.; Jarvis, M. C. Comparative structure and biomechanics of plant primary and secondary cell walls. Front. Plant Sci. 2012, 3, 204.

(34) Szymańska-Chargot, M.; Chylińska, M.; Pieczywek, P. M.; Rösch, P.; Schmitt, M.; Popp, J.; Zdunek, A. Raman imaging of changes in the polysaccharides distribution in the cell wall during apple fruit development and senescence. Planta 2016, 243 (4), 935-945.

(35) Lattanzi, F. A.; Ostler, U.; Wild, M.; Morvan-Bertrand, A.; Decau, M. L.; Lehmeier, C. A.; Meuriot, F.; Prud'homme, M. P.; Schäufele, R.; Schnyder, H. Fluxes in central carbohydrate metabolism of source leaves in a fructan-storing $\mathrm{C} 3$ grass: rapid turnover and futile cycling of sucrose in continuous light under contrasted nitrogen nutrition status. J. Exp. Bot. 2012, 63 (6), 2363-2375.

(36) Janssen, B. J.; Thodey, K.; Schaffer, R. J.; Alba, R.; Balakrishnan, L.; Bishop, R.; Bowen, J. H.; Crowhurst, R. N.; Gleave, A. P.; Ledger, S.; McArtney, S.; Pichler, F. B.; Snowden, K. C.; Ward, S. Global gene 
expression analysis of apple fruit development from the floral bud to ripe fruit. BMC Plant Biol. 2008, 8, 16.

(37) Zhu, Z.; Liu, R.; Li, B.; Tian, S. Characterization of genes encoding key enzymes involved in sugar metabolism of apple fruit in controlled atmosphere storage. Food Chem. 2013, 141, 3323-3328.

(38) Shangguan, L.; Song, C.; Leng, X.; Kayesh, E.; Sun, X.; Fang, J. Mining and comparison of the genes encoding the key enzymes involved in sugar biosynthesis in apple, grape, and sweet orange. Sci. Hortic. 2014, 165, 311-318.

(39) Matros, A.; Peshev, D.; Peukert, M.; Mock, H. P.; Van den Ende, W. Sugars as hydroxyl radical scavengers: proof-of-concept by studying the fate of sucralose in Arabidopsis. Plant J. 2015, 82 (5), $822-839$.

(40) Kahlau, S.; Bock, R. Plastid transcriptomics and translatomics of tomato fruit development and chloroplast-to-chromoplast differentiation: chromoplast gene expression largely serves the production of a single protein. Plant Cell 2008, 20, 856-874.

(41) Asgher, M.; Khan, N. A.; Khan, M. I.; Fatma, M.; Masood, A. Ethylene production is associated with alleviation of cadmium-induced oxidative stress by sulfur in mustard types differing in ethylene sensitivity. Ecotoxicol. Environ. Saf. 2014, 106, 54-61.

(42) Masood, A.; Iqbal, N.; Khan, N. A. Role of ethylene in alleviation of cadmium-induced photosynthetic capacity inhibition by sulphur in mustard. Plant, Cell Environ. 2012, 35 (3), 524-533.

(43) Rouhier, N.; Cerveau, D.; Couturier, J.; Reichheld, J. P.; Rey, P. Involvement of thiol-based mechanisms in plant development. Biochim. Biophys. Acta, Gen. Subj. 2015, 1850 (8), 1479-1496.

(44) Cheng, M. C.; Liao, P. M.; Kuo, W. W.; Lin, T. P. The Arabidopsis ETHYLENE RESPONSE FACTOR1 regulates abiotic stress-responsive gene expression by binding to different cis-acting elements in response to different stress signals. Plant Physiol. 2013, 162 (3), 1566-1582.

(45) Kumar, D.; Hazra, S.; Datta, R.; Chattopadhyay, S. Transcriptome analysis of Arabidopsis mutants suggests a crosstalk between $\mathrm{ABA}$, ethylene and GSH against combined cold and osmotic stress. Sci. Rep. 2016, 6, 36867.

(46) Liu, W.; Stewart, C. N., Jr. Plant synthetic biology. Trends Plant Sci. 2015, 20 (5), 309-317.

(47) Bai, Y.; Dougherty, L.; Cheng, L.; Zhong, G. Y.; Xu, K. Uncovering co-expression gene network modules regulating fruit acidity in diverse apples. BMC Genomics 2015, 16, 612.

(48) Moschen, S.; Higgins, J.; Di Rienzo, J. A.; Heinz, R. A.; Paniego, N.; Fernandez, P. Network and biosignature analysis for the integration of transcriptomic and metabolomic data to characterize leaf senescence process in sunflower. BMC Bioinf. 2016, 17 (Suppl 5), 174.

(49) Sun, R.; Chang, Y.; Yang, F.; Wang, Y.; Li, H.; Zhao, Y.; Chen, D.; Wu, T.; Zhang, X.; Han, Z. A dense SNP genetic map constructed using restriction site-associated DNA sequencing enables detection of QTLs controlling apple fruit quality. BMC Genomics 2015, 16, 747.

(50) Kenis, K.; Keulemans, J.; Davey, M. W. Identification and stability of QTLs for fruit quality traits in apple. Tree Genet. Genomes 2008, 4, 647-661.

(51) Bowles, D.; Lim, E. K.; Poppenberger, B.; Vaistij, F. E. Glycosyltransferases of lipophilic small molecules. Annu. Rev. Plant Biol. 2006, 57, 567-597.

(52) Pegoraro, C.; Tadiello, A.; Girardi, C. L.; Chaves, F. C.; Quecini, V.; de Oliveira, A. C.; Trainotti, L.; Rombaldi, C. V. Transcriptional regulatory networks controlling woolliness in peach in response to preharvest gibberellin application and cold storage. BMC Plant Biol. 2015, 15, 279.

(53) Simpson, J. P.; Ohlrogge, J. B. A novel pathway for triacylglycerol biosynthesis is responsible for the accumulation of massive quantities of glycerolipids in the surface wax of bayberry (Myrica pensylvanica). Plant Cell 2016, 28, 248-264.

(54) Etienne, A.; Génard, M.; Lobit, P.; Mbeguié-A-Mbéguié, D.; Bugaud, $\mathrm{C}$. What controls fleshy fruit acidity? A review of malate and citrate accumulation in fruit cells. J. Exp. Bot. 2013, 64 (6), 14511469. 